\title{
Ionomer Films Impact on The Structure, Flow Regime, and The Wettability of The Catalyst Layer of PEMFC
}

\author{
Karrar Alofari ${ }^{1,}{ }^{*}$, Ezequiel Me' dici $^{1}$, Kazuya Tajiri $^{1}$, and Jeffrey Allen ${ }^{1}$ \\ ${ }^{1}$ MTU, ME-EM Department, 49931 Houghton MI, USA
}

\begin{abstract}
Percolation testing and contact angle measurements have been used to investigate the role of relative humidity on structure, mass transport, and wettability of a PEM fuel cell catalyst layer and membrane. Four samples were tested, two catalyst layers and two membranes. Structure and mass transport changes in the catalyst layers resulting from RH changes were studied in terms of percolation pressure. A clear change in the structure between low and high RH conditioning was observed. Relative humidity (RH) cycling also impacted percolation pressures with an indication of catalyst layer cracking. In addition, $\mathrm{RH}$ effect on wettability of both catalyst layers and membranes was studied by measuring contact angles of sessile drops.
\end{abstract}

\section{Introduction}

Ionomer in the catalyst layer form nano-scale thin films $[1,2]$ that often have the same chemistry as the much thicker electrolyte membranes, though a distinctive difference in properties such as water uptake and conductivity are observed $[3,4]$. The chemical structure of the ionomer consists of hydrophilic sulfonic group clusters that work as a protonic path, which are connected by a side chain to a hydrophobic fluorocarbon backbone that keeps the ionomer both mechanically and chemically stable $[5,6]$. Transport properties of the ionomer are affected by hydration $[4,7,8]$. Both thin $(7 \mathrm{~nm}$ $[1])$ and thick (>10m $[9,10])$ ionomer films interact with vapor to alter the orientation of sulfonic acid side chains [5, 11]. This affects wettability and conductivity in the ionomer films $[12,13]$.

The ionic domains in the ionomer retains water, where the number of water molecules per sulfonic acid group can reach up to 8 as in ionomer thin films in the catalyst layer [4], and $15 \mathrm{in}$ electrolyte membrane $[10,14]$ when saturated with vapor. Thus, the pore size distribution of a catalyst layer may be shifted towards a lower average effective radius as the ionomer swells [15, 16]. This will have an impact on reactant transport resistances [17, 18, 19]. Cycling the relative humidity of the catalyst layer results in the absorption/release of water to/from ionomer films, which in turn leads to structural stresses and potential defect (crack) growth [20].

In order to macroscopically probe the effect of $\mathrm{RH}$ on catalyst layers, percolation testing was conducted using a modified Hele-Shaw arrangement. Percolation behaviour and projected wetted area will help investigate the internal structural changes and the flow regimes as the RH condition changes. Similarly, the sessile drop method is adapted to observe the macroscopic changes in the wettability over a range of $\mathrm{RH}$ conditioning.

\section{Experimental Section}

\subsection{Percolation Testing}

Figure 1 illustrates the modified Hele-Shaw approach used for percolation testing. A catalyst layer sample is compressed between two transparent PMMA platens and liquid is injected at a constant rate through a $2 \mathrm{~mm}$ diameter hole located in the middle of the lower platen. $\mathrm{RH}$ conditioning is achieved by injecting gas nitrogen through the test setup prior to liquid injection. $\mathrm{RH}$ conditioning occurred at room temperature $\left(22\right.$ to $\left.25^{\circ} \mathrm{C}\right)$.

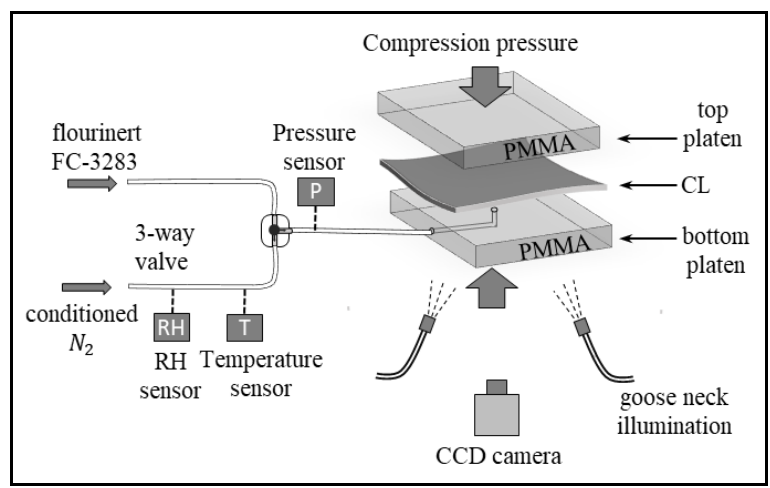

Fig. 1. Percolation setup using a modified Hele-Shaw apparatus.

\footnotetext{
* Corresponding author: ktalofar@mtu.edu
} 
Table 1. Catalyst Layers data.

\begin{tabular}{|c|c|c|c|}
\hline Sample & Pt loading & EW & I/C \\
\hline CL725 & - & 725 & 0.8 \\
\hline CL825 & $0.19 \mathrm{mg} / \mathrm{cm} 2$ & 825 & 0.9 \\
\hline
\end{tabular}

$\mathrm{RH}$ of the nitrogen was measured using a Mega 2560 R3 board that was connected to an Arduino computer. After sufficient RH conditioning, an inert liquid (FC3283 Fluorinert, $3 \mathrm{M}$ ) was injected at a constant flow rate using an ultra-low flow rate syringe pump (Harvard Apparatus, 2274). Injection pressure (i.e., percolation pressure) is measured using a pressure transducer (Omega 209-30V15G10V) located just upstream of the sample injection location. The projected area of the catalyst layer containing liquid was recorded using a CCD camera (Panasonic GP-KS125) at 30 frames per second. Further details of the test apparatus are provided in Alofari et al. [21] and Medici and Allen [22].

Two catalyst layers with equivalent weights of 725 and 825 (provided by $3 \mathrm{M}$ ) were tested over a range of $\mathrm{RH}$ conditioning. Samples were conditioned at low and high RH as shown in Figure 2 for up to three hours. Pertinent material properties for the catalyst layers are listed in Table 1. CL725 and CL825 refer to catalyst layers with equivalent weights (EW) of 725 and 825 , respectively. The ionomer-to-carbon (I/C) ratio for CL725 and CL825 are 0.8 and 0.9, respectively, and CL725 does not include any platinum catalyst.

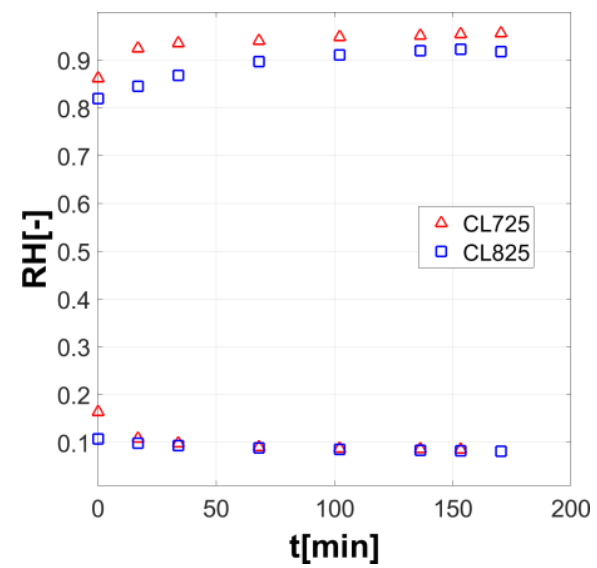

Fig. 2. Measured RH versus time while conditioning the CLs.

\subsection{Contact Angle Measurement}

Wettability of the catalyst layer samples CL725 and CL825 as well as electrolyte membranes with the same chemistry and equivalent weight, PEM725 and PEM 825, respectively, was measured using the sessile drop method. Drop profile images were measured using a long working distance microscope paired with a CCD camera with a uniform backlight. Consistent, accurate, and repeatable drop sizes were deposited using a precision threaded syringe (Hamilton 1750TPLT) in which one plunger rotation is equivalent to $5.27 \mu \mathrm{L}$. Drop size control is illustrated in Figure 3.

Catalyst layer and membrane samples were conditioned

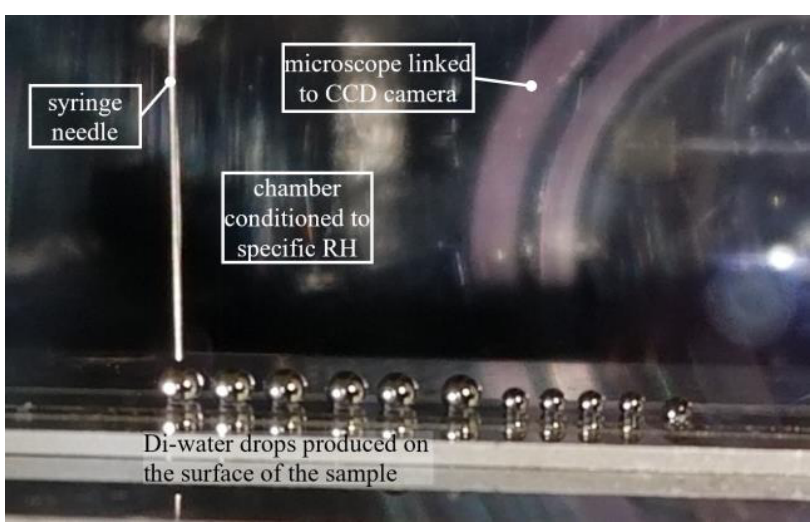

Fig. 3. Water drops on catalyst layer sample for contact angle measurements.

by first drying in a desiccator for two days. Samples were placed into a sealed chamber that enclosed the sample platform. Humidified air flowed through the chamber for a minimum of 60 minutes before drop deposition to ensure constant humidity conditions. The exception was the $0 \% \mathrm{RH}$ samples that did not use conditioned air circulation. RH measurements were the same as for percolation testing. After conditioning, multiple drops of deionized water were placed on the catalyst layer or membrane surface and imaged.

Contact angles were determined by fitting a Laplace curve to the drop profile, then intersecting the curve at the substrate location. The static contact angle is determined by the value of the Laplace curve at the intersection. For each measurement, a minimum of three independent images were captured within 5 seconds to minimize effects of evaporation. The reported contact angle is the average of the three contact angles and uncertainty is related to the standard deviation.

The rough, heterogeneous surfaces of catalyst layers results in a drop size dependence of the measured contact angle [23, 24]. Therefore, drop deposition volume was carefully controlled for consistency with $3.96 \mu \mathrm{L}$ for catalyst layers and $1.32 \mu \mathrm{L}$ for membranes.

\section{Results and Discussion}

\subsection{Percolation Test Results}

Table 2 shows the details of the RH conditioning and percolation flow rate for each test. Transient percolation pressure changes dramatically with $\mathrm{RH}$ conditioning, indicating structural changes in the catalyst layer. Figure 4a shows percolation pressure at low $(9 \%)$ and high (95\%) RH conditioning for CL725. The injection rate of

Table 2. RH Conditioning and Percolation Flow Rate.

\begin{tabular}{|c|c|c|c|}
\hline Sample & Sample Size & RH \% & $\begin{array}{c}\text { Injection } \\
\text { Flow Rate }\end{array}$ \\
\cline { 1 - 1 } CL725 & \multirow{3}{*}{$3.8 \mathrm{~cm} \times 3.8 \mathrm{~cm}$} & 9 & \multirow{2}{*}{$\begin{array}{c}0.396 \\
\mathrm{~mm} 3 / \mathrm{min}\end{array}$} \\
\cline { 1 - 1 } CL725 & & 95 & \\
\cline { 1 - 1 } CL825 & & 92 & \\
\hline CL825 & &
\end{tabular}




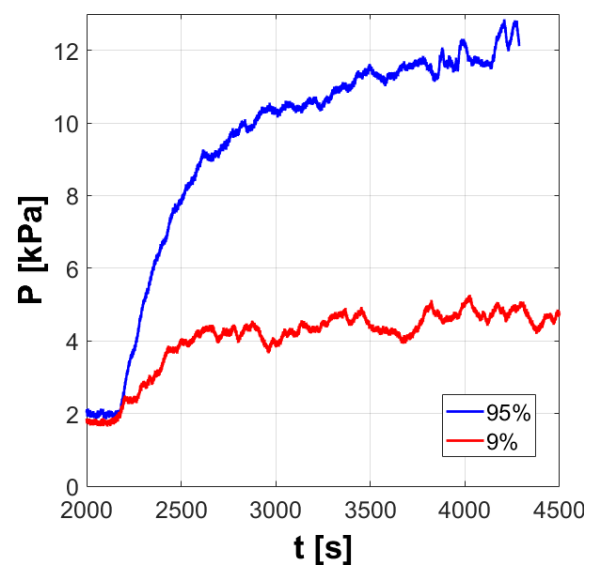

(a) CL725

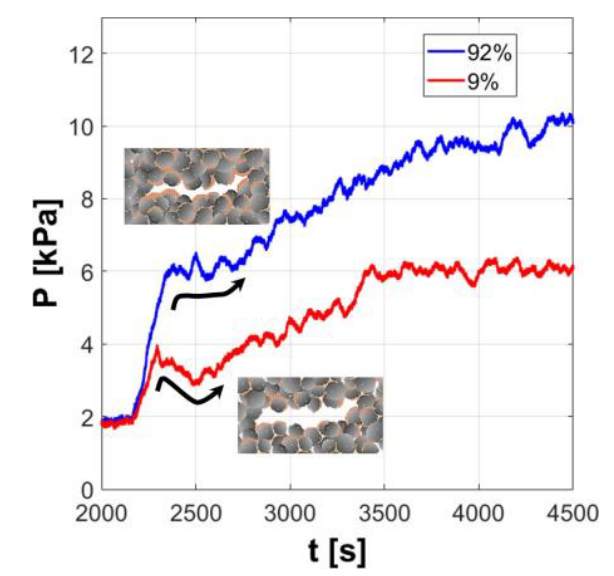

(b) CL825

Fig. 4. Percolation pressure at low and high RH conditioning at constant injection rate of $0.396 \mathrm{~mm} 3 / \mathrm{min}$ for (a) CL725 and (b) CL825.

FC3283 was $0.396 \mathrm{~mm} 3 / \mathrm{min}$ for all tests shown. The percolation pressure curve for CL725 at 9\% condition levels off after approximately 2500 seconds, whereas the $95 \%$ test exhibits a continuous rise at much higher pressures. The difference in percolation pressure is likely due to ionomer swelling resulting in a smaller average pore size. The result is an increase in mass transport resistance within the catalyst layer at high $\mathrm{RH}$ conditioning. A similar effect is observed in CL825, which had a defect (crack) in the catalyst layer. The instant FC3283 encountered this defect is easily detected in the transient percolation pressure as shown in Figure 4b. After the defect, or crack, fills, then the pressure curves exhibit a similar trend as for the CL725 sample.

Transient percolation pressure data in Figure $4 \mathrm{a}$ indicate that imbibition changes with $\mathrm{RH}$ conditioning. At $9 \%$ conditioning, the pressure curves exhibit a classic capillary fingering response and at $95 \%$ the pressure curves exhibit a stable displacement response [22]. In stable displacement, viscous effects dominate the advancing liquid front and liquid is invading most, if not all, pores. For a fixed flow rate, the injection pressure continuous to increase as the liquid advances. In capillary fingering, at the liquid-gas interface, fingers form with a nearly levels off percolation pressure to a constant value. The stable displacement

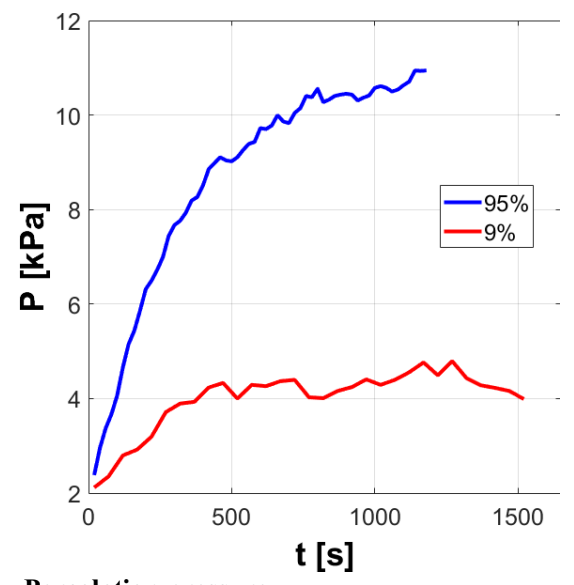

(a) Percolation pressure.

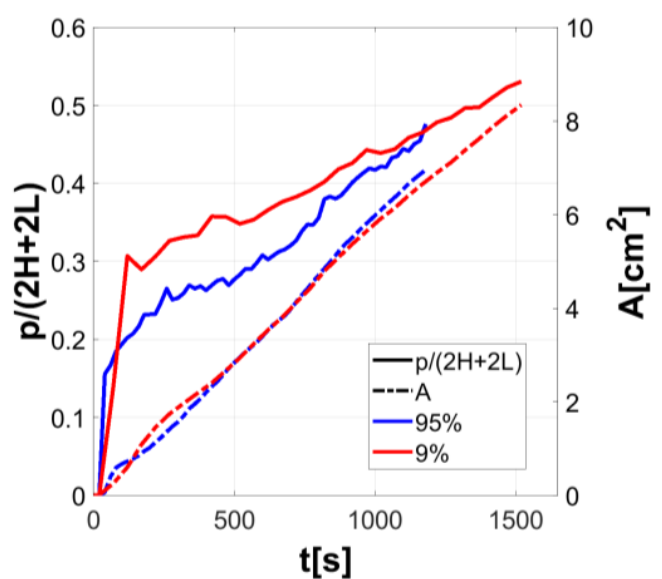

(b) Wetted perimeter and projected area.

Fig. 5. The data include the beginning of the percolation of the liquid phase until the break through. (a) Transient percolation pressure and (b) scaled wetted area and wetted perimeter for CL725 at an injection rate of $0.396 \mathrm{~mm} 3 / \mathrm{min}$. The perimeter of the wetted area, $\mathrm{P}$, is scaled by the perimeter of the catalyst layer sample, $2 \mathrm{H}+2 \mathrm{~L}$.

and capillary fingering response is reinforced in the imaging data. Figure 5a compares the injection pressure for CL725 at high and low RH conditioning with wetted area and perimeter data for the same tests in Figure 5b. The wetted area follows the same trend, is the same for high and low RH conditioning, which is expected for a constant rate of injection. The rate that they advanced is not the same, though with the $95 \%$ RH test ending earlier than the $9 \% \mathrm{RH}$ test. A percolation test ends when liquid reaches the edge of the catalyst layer sample. The wetted area results are indicative of a smaller average pore size for the high RH sample as compared to the low RH sample. There is less overall void volume in the high RH sample, so at a constant rate of injection the test will end earlier.

The scaled perimeter (perimeter of the projected wetted area divided by perimeter of sample) in Figure 5b, like the pressure response, indicates a difference in imbibition flow regime. A dry catalyst layer, such as the $9 \%$ RH sample, has a relatively large average pore size that more readily facilitates capillary fingering. In contrast, the relatively small average pore size of the wet catalyst layer (95\% RH sample), resulting from the 
hypothesized ionomer swelling, increases the local viscous stresses at the advancing liquid-vapor interface resulting in stable displacement.

The hypothesized shift in average pore size due to ionomer swelling in the catalyst layer is supported by varying measured water uptakes with RH. Figure 6 shows the water content, $\lambda$, of nafion for thick membranes [14, 25] and for ultrathin ionomer films with similar equivalent weight as CL725 [4]. As RH is increased, more water retained by hydrophilic sulfonic acid clusters in the ionomer, which results in internal structural changes. As RH decreases, the absorbed water is released. Water uptake and release with RH is repeatable, but the cyclic effect on catalyst layer structure might not be.

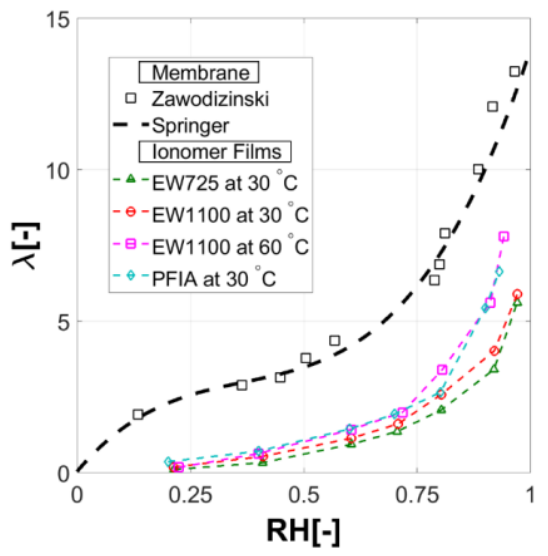

Fig. 6. RH effect on the water content $(\lambda)$ in the hydrophilic sulfonic acid side chain clusters for thick nafion membrane [ 14 , $25]$ and ultra-thin ionomer films [4].

\subsection{RH Effects on Wettability}

Sessile drop contact angle measurements were conducted on catalyst layers (CL725 and CL825) and membranes (PEM725 and PEM825) for a range of RH conditioning, see Figure 7. For both catalyst layer and membrane samples the static contact angle increased then decreased with increasing $\mathrm{RH}$ conditioning. The lowest values of contact angle for the catalyst layers occurs at $0 \% \mathrm{RH}$ conditioning. This is also where there is minimal difference between CL725 and CL825, less than 1० and within the measurement uncertainty.

For both catalyst layers, as RH increases from $0 \%$, there is an immediate difference in static contact angle that can likely be attributed to the dispersed Pt catalyst particles present in the CL825 sample, as shown in Figure $7 \mathrm{a}$, where the maximum static contact angle that occurs is approximately 50 to $60 \% \mathrm{RH}$. As RH is further increased the static contact angle decreases. The difference in the $\mathrm{I} / \mathrm{C}$ ratio between the two catalyst layers does not appear to have any significant effect since the trends are the same.

Despite good repeatability, changes in static contact angle for the heterogeneous surfaces of these catalyst layers are difficult to explain. Based on preliminary results from laser surface profiling there is no measurable change in surface roughness $(0.29 \mu \mathrm{m} \pm 0.37$ for CL725, and $0.75 \mu \mathrm{m} \pm 0.93$ for CL825) with RH conditioning. Our hypothesis is sulfonic acid side chains become less exposed at the catalyst layer surface as the ionomer films take up additional water as $\mathrm{RH}$ conditioning increases. For RH conditions above 50-60\%, morphological alterations associated with the non-linear increase in water uptake, shown in Figure 6, there is a decrease in the catalyst layer's surface hydrophobicity.

Figure $7 \mathrm{~b}$ shows the static contact angle measurements for the membrane samples PEM725 and PEM825. The static contact angles are approximately $50^{\circ}$ less than those on the catalyst layer. This is largely due to the absence of surface roughness on the membranes so there is no structural component to the contact angle measurement. There is a slight increase in static contact angle with increased $\mathrm{RH}$ conditioning from $0 \%$ to $25 \%$. Unlike the catalyst layer samples, static contact angles are relatively constant with increased $\mathrm{RH}$ conditioning. The exception is at the highest RH of $90 \%$ for which an absorbed water film may have begun to form on the membrane surface. PEM725 exhibits overall larger contact angles as compared to PEM825, which was unexpected, though this is consistent with the results of water content in the ionomer films shown in Figure 6, where the calculated $\lambda$ at $30{ }^{\circ} C$ for EW725 is less than EW1100. With higher the EW there are less sulfonic acid groups (hydrophilic part) and less water content [26].

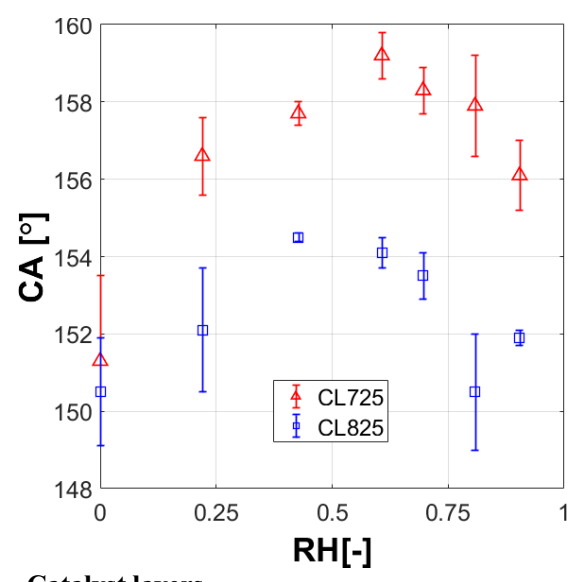

(a) Catalyst layers

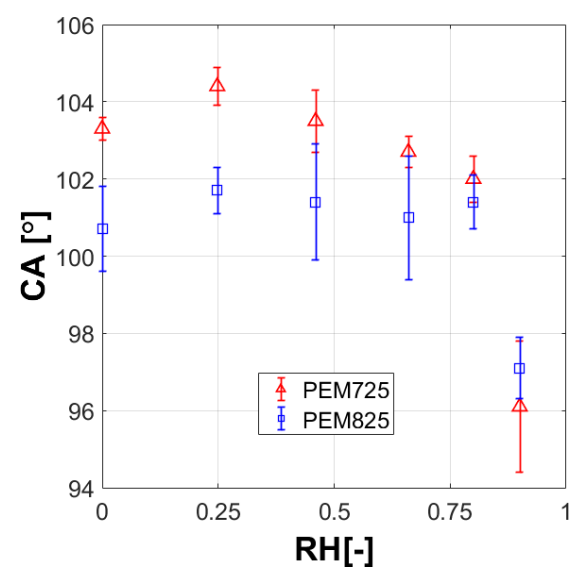

(b) Membranes

Fig. 7. Static contact angles on (a) catalyst layer samples and (b) membranes for range of $\mathrm{RH}$ conditioning. 
There is a possibility of changes in the chemical structure orientation in the membrane similar to the observations of Bass et al. [5].

\section{Conclusion}

Changes in mass transport resistance and wettability of catalyst layers and membranes were studied using macroscopic observations of percolation and contact angle testing. Percolation pressure and liquid front dynamics for low and high $\mathrm{RH}$ conditioning indicate structural changes are occurring in the catalyst layer samples. These changes are significant enough to alter the imbibition flow regime for the same liquid injection rates. Static contact angle measurements on the catalyst layer surfaces also indicate possible morphological changes.

\section{Acknowledgement}

This work was supported in part by The Department of Energy, Office of Energy Efficiency and Renewable Energy (EERE) under award number DE-EE0007650. Additional support was provided by the Higher Committee for Education Development (HCED) of the Iraq Ministry of Higher Education, the Department of Mechanical Engineering - Engineering Mechanics at Michigan Tech, and funds from the John F. and Joan M. Calder Professorship. The authors thank Dr. Andrew Haug for supply the test samples and Dr. Kevin Cooper of Scribner Associates, Inc. for providing a gas humidifier for sample conditioning.

\section{References}

1. Lopez-Haro, M., Guétaz, L., Printemps, T., Morin, A., Escribano, S., Jouneau, P.H., Bayle-Guillemaud, P., Chandezon, F. and Gebel, G., Nature communications, 5, 1 (2014).

2. Paul, D. K., Karan, K., Docoslis, A., Giorgi, J. B., and Pearce, J. Macromolecules, 46, 9 (2013).

3. Paul, D. K., McCreery, R., and Karan, K. Journal of the electrochemical society, 161,14 (2014).

4. Shrivastava, U. N., Fritzsche, H., and Karan, K. Macromolecules, 51,23 (2018).

5. Bass, M., Berman, A., Singh, A., Konovalov, O., and Freger, V., Macromolecules, 44, 8 (2011).

6. Weber, A. Z. and Newman, J.. Journal of the Electrochemical Society, 151, 2 (2004).

7. Shrivastava, U. N., Suetsugu, K., Nagano, S., Fritzsche, H., Nagao, Y., and Karan, K., Soft Matter, 16, 5 (2020).

8. Nguyen, H.-D., Porihel, R., Brubach, J.-B., Planes, E., Soudant, P., Judeinstein, P., Porcar, L., Lyonnard, S., and Iojoiu, C., ChemSusChem, 13, 3 (2020).

9. Gierke, T.D., Munn, G.E. and Wilson, F., Journal of Polymer Science: Polymer Physics Edition, 19, 11 (1981).
10. He, Q., Kusoglu, A., Lucas, I.T., Clark, K., Weber, A.Z. and Kostecki, R., The Journal of Physical Chemistry B, 115, 40 (2011).

11. Van Nguyen, T., Nguyen, M. V., Lin, G., Rao, N., Xie, X., and Zhu, D.-M., Electrochemical and Solid State Letters, 9, 2 (2005).

12. Paul, D. K. and Karan, K., The Journal of Physical Chemistry C, 118, 4 (2014).

13. Bass, M., Berman, A., Singh, A., Konovalov, O., and Freger, V., The Journal of Physical Chemistry B, 114, 11 (2010).

14. Zawodzinski Jr, T. A., Neeman, M., Sillerud, L. O., and Gottesfeld, S., The Journal of Physical Chemistry, 95, 15(1991).

15. Kusoglu, A., Kushner, D., Paul, D. K., Karan, K., Hickner, M. A., and Weber, A. Z., Advanced Functional Materials, 24, 30 (2014).

16. Ahangar, S. B., Bellur, K., Medici, E., Tajiri, K., Allen, J. S., and Choi, C. K., ECS Transactions, 92, 8 (2019).

17. Wang, G., Osmieri, L., Star, A. G., Pfeilsticker, J., and Neyerlin, K. C., Journal of The Electrochemical Society, 167, 4 (2020).

18. Zhang, F.-Y., Spernjak, D., Prasad, A. K., and Advani, S. G., Journal of The Electrochemical Society, 154, 11 (2007).

19. Deevanhxay, P., Sasabe, T., Tsushima, S., and Hirai, S., Electrochemistry communications, 22 (2012).

20. Chang, Y., Liu, J., Li, R., Zhao, J., Qin, Y., Zhang, J., Yin, Y., and Li, X., Energy Conversion and Management, 189 (2019).

21. Alofari, K. T., Medici, E., Tajiri, K., and Allen, J. S., ECS Transactions, 92, 8 (2019).

22. Medici, E. and Allen, J. (2009). Journal of Power Sources, 191(2):417- 427.

23. Drelich, J. and Miller, J. D. Journal of colloid and interface science, 164, 1 (1994).

24. Wang, X., Wang, W., Qu, Z., Ren, G., and Wang, H., International Journal of Hydrogen Energy, (2021).

25. Springer, T. E., Zawodzinski, T., and Gottesfeld, S. Journal of the electrochemical society, 138, 8 (1991).

26. Ramaswamy, N., Kumaraguru, S., Koestner, R., Fuller, T., Gu, W., Kariuki, N., Myers, D., Dudenas, P. J., and Kusoglu, A. Journal of The Electrochemical Society, 168, 2 (2021). 